\title{
EVALUACIÓN DE SEIS SISTEMAS DE SANGRÍA PARA CUATRO CLONES DE HEVEA BRASILIENSIS (WILLD. EX ADR. DE JUSS) MUELL. ARG., EN LA ALTILLANURA COLOMBIANA
}

\author{
Assessment of six tapping systems for four Hevea brasiliensis \\ (Willd. Ex Adr. De Juss) Muell. Arg., clones in the Colombian High Plains
}

Palabras clave: Hevea brasiliensis, sangría, producción, Ethefon, caucho natural.

Keywords: Hevea brasiliensis, tapping, Ethephon, yield, natural rubber.
Isaac Ouesada Méndez:

Fabio Ancízar Aristizábal Gutiérrez

Dolly Montoya Castaño ${ }^{3}$

Bernardo Chaves Córdoba

\section{RESUMEN}

Este trabajo evaluó el desempeño productivo de cuatro clones de caucho natural (Hevea brasiliensis [Willd. ex Adr. de Juss.] Muell.-Arg.), sometidos a seis diferentes sistemas de sangría, los cuales incluyeron la combinación de dos diferentes frecuencias de sangrado cada cuatro y cinco días $(\mathrm{d} / 4$ y d/5) y cuatro concentraciones de Ethefon $(0 \%$, $2.5 \%, 3.0 \%$ y $5 \%$ ) con un número de aplicaciones entre 4 a 8 veces por año. Los ensayos se realizaron en la plantación de la empresa MAVALLE S.A., ubicada en la altillanura colombiana, bajo un diseño de bloques completos al azar con cuatro repeticiones. Se obtuvieron producciones promedio por año de $4446 \mathrm{~g}$ de caucho/árbol para el clon RRIM 600 en un sistema $1 / 2 \mathrm{~S}, \mathrm{~d} / 4,6 \mathrm{~d} / 7,10 \mathrm{~m} / 12$, ет $2.5 \%$, Pa $7 / \mathrm{y}$; $2696 \mathrm{~g}$ de caucho/árbol para el clon PB 260 bajo un sistema $1 / 2 \mathrm{~S}, \mathrm{~d} / 4,6 \mathrm{~d} / 7,10 \mathrm{~m} / 12$, ет $2.5 \%$, Pa $5 / \mathrm{y}$; $3822 \mathrm{~g}$ de caucho/árbol para el clon IAN 873 en un sistema $1 / 2 \mathrm{~S}, \mathrm{~d} / 4,6 \mathrm{~d} / 7,10 \mathrm{~m} / 12$, ет $3.3 \%$, Pa $8 / \mathrm{y}$, y $3472 \mathrm{~g}$ de caucho/árbol para el clon GT 1 bajo una sistema $1 / 2 \mathrm{~S}, \mathrm{~d} / 4,6 \mathrm{~d} / 7,10 \mathrm{~m} / 12$, Ет $2.5 \%$, Pa $5 / \mathrm{y}$. Se obtuvo la mayor producción en una frecuencia de sangrado cada cuatro días para los clones RRIM 600, GT 1 у РB 260 .

\begin{abstract}
The rubber yield of four Hevea brasiliensis (Willd. ex Adr. de Juss.) Muell.-Arg. clones was evaluated on the Colombian high plains in the Meta department: IAN 873 and RRIM 600 (third year of tapping being done) and PB 260 and GT 1 (first year of tapping). Six tapping systems were used, including a combination of different tapping frequencies $(\mathrm{d} / 4$ and $\mathrm{d} / 5)$, Ethephon concentrations ( $0 \%, 2.5 \%, 3.3 \%$ and $5 \%)$ and a number of applications per year (4 to 8 ) were also used depending on the clone. The production figures for one commercial year were obtained from assays, using a completely randomblock design (having four repetitions) independently defined for each clone: having on average 4446 $\mathrm{g}$ rubber/tree of produce per year for the RRIM 600 clone within a system $1 / 2 \mathrm{~S}, \mathrm{~d} / 4,6 \mathrm{~d} / 7,10 \mathrm{~m} / 12$, ET $2.5 \%, \mathrm{~Pa} 7 / \mathrm{y} ; 2696 \mathrm{~g}$ rubber/tree for the PB 260 clone with $1 / 2 \mathrm{~S}, \mathrm{~d} / 4,6 \mathrm{~d} / 7,10 \mathrm{~m} / 12$, ET $2.5 \%$, Pa $5 / \mathrm{y}$; $3822 \mathrm{~g}$ rubber/tree for the IAN 873 clone, $1 / 2 \mathrm{~S}, \mathrm{~d} / 4$, $6 \mathrm{~d} / 7,10 \mathrm{~m} / 12$, ET $3.3 \%, \mathrm{~Pa} 8 / \mathrm{y}$, and $3472 \mathrm{~g}$ rubber/ tree for the GT 1 clone, $1 / 2 \mathrm{~S}, \mathrm{~d} / 4,6 \mathrm{~d} / 7,10 \mathrm{~m} / 12$, ET $2.5 \%$, Pa 5/y. The highest produce was obtained with a four-day tapping frequency.
\end{abstract}

1 Instituto de Biotecnología, Universidad Nacional de Colombia (IBUN), Grupo de Bioprocesos y Bioprospección (línea de investigación en Caucho Natural). Correspondencia: iquesadam@unal.edu.co

2 Departamento de Farmacia, Facultad de Ciencias, Universidad Nacional de Colombia.

3 Instituto de Biotecnología, Universidad Nacional de Colombia (IBUN).

4 Facultad de Agronomía, Universidad Nacional de Colombia. 


\section{INTRODUCCIÓN}

El género Hevea, cuyo nombre proviene de la palabra HYÉvÉ o HEVÉ , que significa "árbol que llora" o "lagrimas de árbol", es originario de las planicies del Amazonas y la Orinoquia y se encuentra en estado silvestre en Bolivia, Perú, Colombia y los estados de Matto Grosso y Marrano en Brasil (Compagnon 1998).

Según Torres (1984), el primer ensayo del cultivo del árbol de caucho natural con fines industriales en Colombia se realizó en 1910 por el inglés Reynords en las orillas del río Mira, en la finca $\mathrm{La}$ Victoria en el litoral Pacífico. En el año 1943 el Departamento de Agricultura de los Estados Unidos promovió el cultivo tecnificado del árbol del caucho por medio de la instalación de 500 ha en el departamento de Antioquia, de las cuales para la fecha quedaban menos de 150 ha. Fue hasta el año de 1964 que el Instituto Colombiano de la Reforma Agraria (INCORA) desarrolló un plan de fomento basado en las recomendaciones técnicas de Sorensen, Lawsen, Mannis, Langford de Labarre y Bonnaire: se sembraron 400 ha repartidas entre Maguaré (75 ha) y La Mono (325 ha) en el departamento del Caquetá.

En el año 2008, según datos suministrados por la Secretaría Técnica de la Cadena Productiva del Caucho y su Industria, Colombia contaba con alrededor de 30.356 ha plantadas con caucho natural y se proyecta que para el año 2011 se hayan plantado 19.700 nuevas ha. El mayor desarrollo heveicola en Colombia se encuentra en el departamento del Caquetá con un 25\%, seguido por Santander con un $21 \%$ y Antioquia y Meta con un 13\% cada uno (Ministerio de Agricultura y Desarrollo Rural 2008).

De acuerdo con el estudio de zonificación elaborado en el año 1997 por la Corporación Nacional de Investigación y Fomento Forestal (CONIF), Colombia cuenta actualmente con 12.998.944 ha aptas para el cultivo del caucho, de las cuales 4.584.474 están clasificadas como zona de escape al mal suramericano de la hoja o SALB (Souht American Leaf Bligh) - principal limitante de este cultivo- y son favorables para plantaciones con materiales de origen asiático de alta productividad, pero susceptibles al SALB. Las otras 8.144.520 ha están clasificadas como de no escape el uso de material americano con resistencia al SALB.

Posterior a la etapa improductiva del cultivo, en donde las labores culturales están orientadas al control de malezas, la fertilización y el control fitosanitario, el inicio del sangrado de los árboles pone al cultivador a enfrentar el reto de efectuar tanto económica como fisiológicamente un aprovechamiento óptimo de los árboles. Independiente del potencial genético, la capacidad de producción de un clon está determinada principalmente por dos factores propios del proceso de sangrado: la capacidad de regeneración y el tiempo de escurrimiento. Entre más prolongado el tiempo de escurrimiento mayor será la producción y así mismo mayor será el desgaste fisiológico del sistema laticífero del árbol, haciéndose necesario un mayor tiempo para su regeneración. Tradicionalmente el aprovechamiento incluye: i) el uso de bajas frecuencias de sangrado, a fin de proporcionar mayor tiempo de regeneración y ii) el uso de Ethrel ${ }^{\circledR}$ como estimulante, aumentado así el escurrimiento y metabolismo del árbol.

Los sistemas de sangría buscan un conjunto de beneficios que no sólo incluyen el incremento en la producción de látex, sino también la disminución del consumo de corteza y de daños del panel de sangría para lograr mayor vida útil del árbol, mayor tiempo de regeneración del sistema laticífero y menor uso de mano de obra, mejorando así la rentabilidad del cultivo. Lo anterior se logra a través de la selección de la mejor combinación de frecuencias de sangrado, concentración y número anual de aplicaciones del estimulante , manteniendo siempre una alta productividad (D'Auzac et al. 1989).

Escasos son los reportes sobre el desempeño productivo de clones de $H$. brasiliensis en nuestro país, donde sobresale lo reportado por el Centro Nacional de Investigaciones de Café (CENICAFE). Se evaluaron tres clones de origen americano y diez de origen asiático en las condiciones de la zona ca- 
fetera colombiana, en la estación experimental de Paraguacito, municipio de Buenavista, en el departamento del Quindío, y se obtuvieron producciones promedio por año superiores a $4 \mathrm{~kg} /$ caucho/árbol para el clon FX 3864 (López, 2003).

El presente trabajo tuvo como objetivo evaluar la producción de caucho seco de los clones RRIM 600, PB 260 y GT 1 de origen asiático y el clon IAN 873 de origen americano, sometidos a seis sistemas de sangría en la altillanura colombiana.

\section{METODOLOGÍA}

La investigación se realizó en la plantación de caucho natural de la empresa MAVALLE S.A, ubicada en la inspección de Remolino, municipio de Puerto López, en el departamento del Meta. La plantación se encuentra en la Orinoquia colombiana, en un área fisiográfica de altillanura, en un paisaje de sabanas planas bien drenadas con suelos de textura arcillo-arenosa y arcillo-limos, con profundidades mayores a $2 \mathrm{~m}$, bien drenados y con pendientes entre el $3 \%$ y $10 \%$. La temperatura promedio anual es de $28^{\circ} \mathrm{C}$, que oscila entre $19^{\circ} \mathrm{C}$ y $36^{\circ} \mathrm{C}$, con régimen de precipitación monomodal, un promedio anual de $2200 \mathrm{~mm}$ y 8 meses de precipitación superiores a $100 \mathrm{~mm}$. La humedad relativa promedio anual es de $77 \%$, con una mínima de $60 \%$ y una máxima de $85 \%$; así mismo, cuenta con alrededor de 1600 y 1800 horas de sol al año.

El material vegetal de los clones IAN 873 y RRIM 600 fue plantado en año de 1992; los dos clones restantes, PB 260 y GT 1, se plantaron en el año 1993. Con el fin de determinar el tamaño de la unidad experimental, así como los recursos necesarios para realizar el monitoreo de la producción de sangría comercial durante un año, se llevó a cabo un muestreo exploratorio en el mes de enero de 2003, en el que, sobre material vegetal de RRIM 600, IAN 873, FX 3864 у рв 235, se evaluó la variabilidad en el volumen de látex producido.
En febrero de 2003 se instalaron cuatro ensayos donde se evaluaron de manera independiente los clones IAN 873 y RRIM 600 en tercer año de sangría y los clones Gт 1 у Рв 260 en primer año de sangría. Se utilizó un diseño de bloques completos al azar, con cuatro repeticiones o bloques, seis tratamientos o sistemas de sangría y diez árboles como unidad experimental. El número de árboles en los cuales se registró la producción para cada clon fue de 240, correspondientes a 24 unidades experimentales. En total se utilizaron 960 árboles para los cuatro clones analizados.

La producción se evaluó en sistemas de sangría con cortes en media espiral ( $1 / 2 \mathrm{~S})$, efectuados durante siete días por semana $(7 \mathrm{~d} / 7)$ en diez meses del año $(10 \mathrm{~m} / 12)$, variando las frecuencias de sangrado cada cuatro y cinco días (d/4, d/5). Se aplicó solución de agua, Carboximetilcelulosa y Ethel ${ }^{\circledR} 48$ sL, cada $76,61,51,43$ y 38 días, con concentraciones del ingrediente activo de Ethel ${ }^{\circledR}$ en $2.5 \%$ y $3.3 \%$, para los clones RRIM 600 y GT 1, concentraciones de $3.3 \%$ y $5 \%$ para el clon IAN 873 y concentraciones de $2.5 \%$ para el clon PB 260. Los tratamientos para cada uno se presentan en la Tabla 1. Estos tratamientos fueron seleccionados según las recomendaciones efectuadas por Doree (1998), producto de su investigación en la plantación de la empresa MICHELIN en Matto Grosso, Brasil.

Las observaciones se realizaron directamente en campo por medio de la medición del volumen de mililitros de látex (ml/árbol) por cada unidad de muestreo. La labor se iniciaba con el sangrado de los árboles; transcurrida una hora de escurrimiento y con el fin de mantener la producción en estado liquido, se efectuaba la aplicación de $0.5 \mathrm{ml} \mathrm{de} \mathrm{NH}_{3}$ [5\% v/v] por árbol, como agente preservante, y tras un mínimo de cuatro horas de escurrimiento se efectuaba la medición de ml/látex producidos por cada uno de los árboles. 
Tabla 1. Sistemas de sangría evaluados en los clones RRIM 600, IAN 873, GT 1 y PB 260 en el año comercial 2003.

\begin{tabular}{|c|c|c|}
\hline Clon & Tratamiento & $\begin{array}{l}\text { Sistemas } \\
\text { evaluados }\end{array}$ \\
\hline \multirow{6}{*}{ 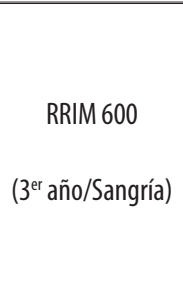 } & $\mathrm{T} 1$ & $\mathrm{~d} / 4^{1}$ sin estimulación (testigo) \\
\hline & $\mathrm{T} 2$ & $\mathrm{~d} / 4$, ET $2.5 \%, 6 / \mathrm{y}^{3}$ \\
\hline & $\mathrm{T} 3$ & $\mathrm{~d} / 4$, ET $2.5 \% 7 / \mathrm{y}$ \\
\hline & $\mathrm{T} 4$ & $\mathrm{~d} / 5^{2}$, sin estimulación (testigo) \\
\hline & T5 & $\mathrm{d} / 5$, ET 3.3\%,7/y \\
\hline & T6 & $\mathrm{d} / 5$, ET $3.3 \%, 8 / \mathrm{y}$ \\
\hline \multirow{6}{*}{$\begin{array}{c}\text { IAN } 873 \\
\text { (3r año/Sangría) }\end{array}$} & $\mathrm{T} 1$ & $\mathrm{~d} / 4$ sin estimulación (testigo) \\
\hline & $\mathrm{T} 2$ & $\mathrm{~d} / 4$, ET $3.3 \%, 7 / y$ \\
\hline & $\mathrm{T} 3$ & $\mathrm{~d} / 4$, ET $3.3 \%, 8 / \mathrm{y}$ \\
\hline & $\mathrm{T} 4$ & $\mathrm{~d} / 5$, sin estimulación (testigo) \\
\hline & T5 & $\mathrm{d} / 5$, ET $5.0 \%, 7 / \mathrm{y}$ \\
\hline & T6 & d/5, ET $5.0 \%, 8 / y$ \\
\hline \multirow{6}{*}{$\begin{array}{c}\text { PB } 260 \\
\text { (1 } 1^{\text {er }} \text { año/Sangría) }\end{array}$} & $\mathrm{T} 1$ & $\mathrm{~d} / 4$, sin estimulación (testigo) \\
\hline & $\mathrm{T} 2$ & $\mathrm{~d} / 4$, ET $2.5 \%, 4 / y$ \\
\hline & $\mathrm{T} 3$ & $\mathrm{~d} / 4$, ET $2.5 \% 5 / \mathrm{y}$ \\
\hline & $\mathrm{T} 4$ & $\mathrm{~d} / 5$, sin estimulación (testigo) \\
\hline & $\mathrm{T} 5$ & $\mathrm{~d} / 5$, ET $2.5 \%, 5 / y$ \\
\hline & T6 & $\mathrm{d} / 5$, ET $2.5 \% 6 / \mathrm{y}$ \\
\hline \multirow{6}{*}{ (1er año/Sangría) } & $\mathrm{T} 1$ & $\mathrm{~d} / 4$, sin estimulación (testigo) \\
\hline & $\mathrm{T} 2$ & $\mathrm{~d} / 4$, ET $2.5 \%, 4 / \mathrm{y}$ \\
\hline & $\mathrm{T} 3$ & $\mathrm{~d} / 4$, ET $2.5 \%, 5 / \mathrm{y}$ \\
\hline & $\mathrm{T} 4$ & $\mathrm{~d} / 5$, sin estimulación (testigo) \\
\hline & $\mathrm{T} 5$ & d/5, ET 3.3\%, 5/y \\
\hline & T6 & d/5, ET 3.3\%, 6/y \\
\hline
\end{tabular}

1: d/4: Frecuencia de sangría cada cuatro días.

2: d/5: Frecuencia de sangría cada cinco días.

3: ET 2.5\%, 6/y: Estimulación con Ethefon al 2.5\%, 6 veces al año.

Como el estudio buscaba determinar la producción promedio en $\mathrm{g}$ de caucho seco por árbol ( $\mathrm{g} / \mathrm{cau}-$ cho/árbol) para los cuatro clones, se llevó a cabo una conversión de los datos de producción ( $\mathrm{ml} / \mathrm{lá}-$ tex/árbol) al valor de caucho seco (g/caucho/árbol) para cada uno de los 960 árboles, utilizando como factores de transformación el contenido de caucho seco (Dry Rubber Content -DRC) y la densidad de látex para cada tratamiento, cuyos valores se presentan en la Tabla 2.

Lo anterior permitió establecer un promedio de producción mensual y anual acumulada por árbol. Utilizando el programa estadístico SAS ${ }^{\circledR}$ (Statistical Analysis System ver. 8) se generó para cada uno de los clones un análisis de varianza (ANAVA), con un grado de significancia de 0.05 .

Con el propósito de determinar las diferencias de los valores de producción promedio anual de g de caucho seco por árbol para cada sistema de sangría, se llevaron a cabo las pruebas de contrastes ortogonales, entre las frecuencias de sangrado d/4 (T1, T2, T3) vs. d/5 (T4, T5, T6), entre el tratamiento sin estimulación y los tratamientos con estimulación de una misma frecuencia de sangrado (T1 vs. T2, T3) y (T4 vs. T5, T6), entre los tratamientos estimulados de una misma frecuencia (T2 vs. T3) y (T5 vs. T6) y entre los tratamientos estimulados (T2, T3 vs. T5, T6) y sin estimulación (T1 vs. T4) de las dos frecuencias. 
Tabla 2. Valores de caucho seco (DRC) para cada uno de los tratamientos evaluados en los cuatro clones.

\begin{tabular}{|c|c|c|c|c|c|c|c|c|}
\hline \multirow{3}{*}{ Tratamiento } & \multicolumn{8}{|c|}{ Clon } \\
\hline & \multicolumn{2}{|c|}{ RRIM 600} & \multicolumn{2}{|c|}{ IAN 873} & \multicolumn{2}{|c|}{ GT 1} & \multicolumn{2}{|c|}{ PB 260} \\
\hline & $\begin{array}{c}\text { Valor de DRC } \\
(\%)^{1} \\
\end{array}$ & Densidad & $\begin{array}{c}\text { Valor de DRC } \\
(\%) \\
\end{array}$ & Densidad & $\begin{array}{c}\text { Valor de DRC } \\
(\%) \\
\end{array}$ & Densidad & $\begin{array}{c}\text { Valor de DRC } \\
(\%) \\
\end{array}$ & Densidad \\
\hline $\mathrm{T} 1$ & 42.69 & 0.976 & 41.25 & 0.973 & 35.10 & 0.971 & 39.17 & 0.978 \\
\hline $\mathrm{T} 2$ & 36.08 & 0.976 & 39.21 & 0.973 & 36.12 & 0.971 & 33.24 & 0.978 \\
\hline $\mathrm{T} 3$ & 37.39 & 0.976 & 39.52 & 0.973 & 34.62 & 0.971 & 36.48 & 0.978 \\
\hline T4 & 43.34 & 0.973 & 43.19 & 0.977 & 39.09 & 0.975 & 37.50 & 0.977 \\
\hline T5 & 40.87 & 0.973 & 40.55 & 0.977 & 36.45 & 0.975 & 27.24 & 0.977 \\
\hline T6 & 39.91 & 0.973 & 41.68 & 0.977 & 3.611 & 0.975 & 38.58 & 0.977 \\
\hline
\end{tabular}

1: El valor está expresado en \% de g/caucho/seco presente en un volumen de $100 \mathrm{ml}$ de látex.

Fuente: Informe final del proyecto "Ajuste y Desarrollo Tecnológico en los procesos de Extracción y Beneficio de Caucho Natural

(Hevea brasiliensis)".

\section{RESULTADOS}

Los resultados del análisis de varianza para cada uno de los clones se presentan en la Tabla 3, de igual forma, los resultados de los contrastes entre los tratamientos evaluados en cada uno de los cuatro clones se presentan en la Tabla 4. Estos resultados se discuten a continuación:

\section{CLON RRIM 600}

El comportamiento de la producción en los diez meses se presenta en la Figura 1, donde se aprecia la mayor producción en los meses de junio, julio y agosto, correspondiente a un $40.4 \%$ de la producción total anual.

El análisis de varianza para valores promedio de producción anual de caucho seco por árbol, de los seis tratamientos evaluados en el clon RRIM 600, presentó diferencias significativas (Tabla 3 ).

Tabla 3. Análisis de varianza del promedio de producción por árbol de los clones RRIM 600, IAN 873, PB 260 y GT 1.

\begin{tabular}{|c|c|c|c|c|c|c|c|c|c|}
\hline \multirow[b]{3}{*}{$\begin{array}{l}\text { Fuente de } \\
\text { variación }\end{array}$} & \multirow[b]{3}{*}{$\begin{array}{c}\text { Grados } \\
\text { de } \\
\text { libertad }\end{array}$} & \multicolumn{8}{|c|}{ Clones } \\
\hline & & \multicolumn{2}{|c|}{ RRIM 600} & \multicolumn{2}{|c|}{ IAN 873} & \multicolumn{2}{|c|}{ PB 260} & \multicolumn{2}{|c|}{ GT 1} \\
\hline & & $\begin{array}{c}\text { Cuadrados } \\
\text { medios }\end{array}$ & $P>F$ & $\begin{array}{l}\text { Cuadrados } \\
\text { medios }\end{array}$ & $P>F$ & $\begin{array}{l}\text { Cuadrados } \\
\text { medios }\end{array}$ & $P>F$ & $\begin{array}{l}\text { Cuadrados } \\
\text { medios }\end{array}$ & $P>F$ \\
\hline $\begin{array}{l}\text { Sistemas de } \\
\text { sangría }\end{array}$ & 5 & 37032230.2 & $0.0003^{*}$ & 6288877.41 & $0.2967^{\mathrm{NS}}$ & 5595024.76 & $<.0001^{*}$ & 15014585.78 & $<.0001^{*}$ \\
\hline Repeticiones & 3 & 15118753.9 & $0.0308^{*}$ & 9655646.73 & $0.1466^{\mathrm{NS}}$ & 651071.49 & $0.1117^{\mathrm{NS}}$ & 4015971.85 & $<.0001^{*}$ \\
\hline $\begin{array}{c}\text { Error } \\
\text { experimental }\end{array}$ & 15 & 3892999.2 & & 4653716.53 & & 274986.92 & & 541277.01 & \\
\hline $\begin{array}{l}\text { Error de } \\
\text { muestreo }\end{array}$ & 216 & 952850.7 & & 1107998.2 & & 305832.7 & & 271339.5 & \\
\hline TOTAL & 239 & & & & & & & & \\
\hline
\end{tabular}

*: Diferencias estadísticas significativas, con probabilidad de $5 \%$.

NS: No existen diferencias estadísticas significativas. 


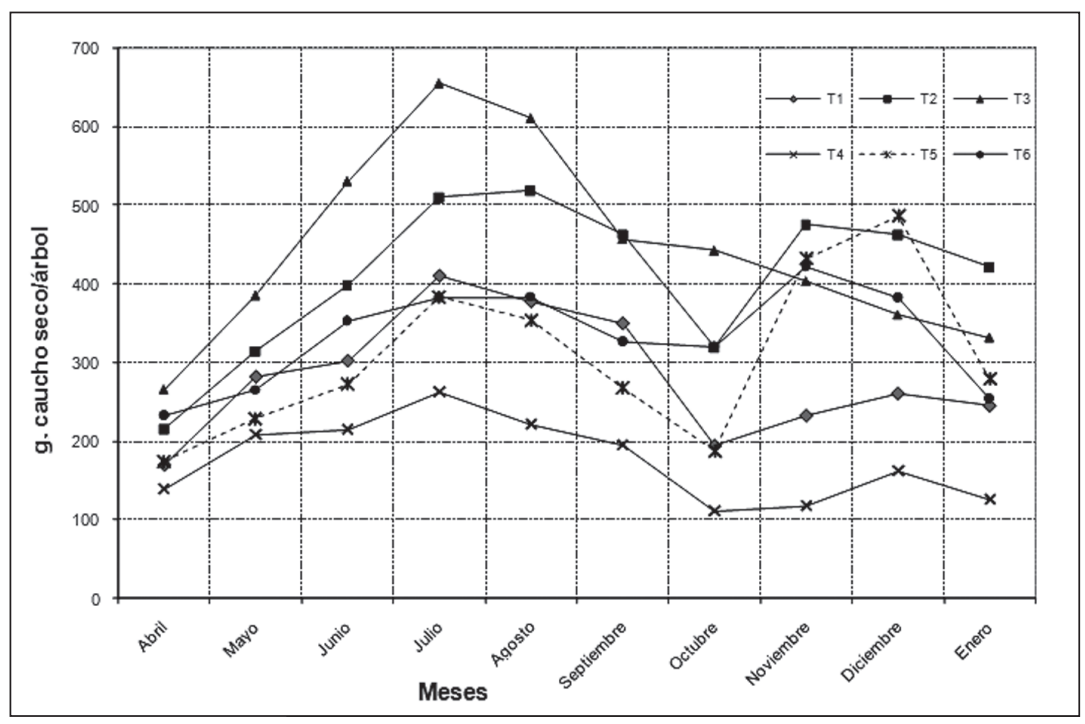

Figura 1. Distribución de la producción promedio mensual de caucho seco por árbol del clon RRRI 600 (año comercial de abril 2003 - enero 2004). T1: Testigo en frecuencia d/4, sin aplicación de estimulante; T2: d/4, ET 2.5\%, 6/y; T3: d/4, ET 2.5\%, 7/y; T4: Testigo en frecuencia d/5, sin aplicación de estimulante; T5: d/5, ET 3.3\%, 7/y, y T6: $\mathrm{d} / 5$, ET $3.3 \%, 8 / \mathrm{y}$.

Tabla 4. Resultados de la prueba de contrastes ortogonales entre los valores promedio de producción de los seis tratamientos evaluados en los clones RRIM 600, PB 260 y GT 1.

\begin{tabular}{|c|c|c|c|c|c|c|c|}
\hline \multirow[b]{3}{*}{ Contraste } & \multirow[b]{3}{*}{$\begin{array}{l}\text { Grados de } \\
\text { libertad }\end{array}$} & \multicolumn{4}{|c|}{ Clones } & & \\
\hline & & \multicolumn{2}{|c|}{ RIMM 600} & \multicolumn{2}{|c|}{ PB 260} & \multicolumn{2}{|c|}{ GT 1} \\
\hline & & $\begin{array}{l}\text { Cuadrados } \\
\text { medios }\end{array}$ & $P>F$ & $\begin{array}{l}\text { Cuadrados } \\
\text { medios }\end{array}$ & $P>F$ & $\begin{array}{l}\text { Cuadrados } \\
\text { medios }\end{array}$ & $P>F$ \\
\hline $\begin{array}{c}\text { T1, T2, T3 vs. T4, } \\
\text { T5, T6 }\end{array}$ & 1 & 69565911.40 & $0.0007^{*}$ & 12143870.29 & $<.0001^{*}$ & 16675256.17 & $<.0001^{*}$ \\
\hline T1 vs. T2 y T3 & 1 & 56609395.59 & $0.0017^{*}$ & 10287135.75 & $0.0295^{*}$ & 30346484.58 & $<.0001^{*}$ \\
\hline T2 vs. T3 & 1 & 2387210.32 & $0.4458^{\text {NS }}$ & 525446.90 & $0.1877^{\mathrm{NS}}$ & 3585682.77 & $0.0212^{*}$ \\
\hline T4 vs. T5 y T6 & 1 & 55228819.89 & $0.0019^{*}$ & 3637727.43 & $0.0024^{*}$ & 24251273.07 & $<.0001^{*}$ \\
\hline T5 vs. T6 & 1 & 1369813.81 & $0.5619^{\mathrm{NS}}$ & 1380943.44 & $0.0406^{*}$ & 214232.29 & $0.5387^{\mathrm{NS}}$ \\
\hline T2 y T3 vs. T5 y T6 & 1 & 46891987.55 & $0.0034^{*}$ & 11397948.42 & $<.0001^{*}$ & 12764137.76 & $0.0002^{*}$ \\
\hline T1 vs. T4 & 1 & 22678184.61 & $0.0290^{*}$ & 1591017.77 & $0.0295^{*}$ & 4081766.84 & $0.0150^{*}$ \\
\hline
\end{tabular}

*: Diferencias significativas (5\%).

NS: No existen diferencias estadísticas significativas. 
Tabla 5. Promedios de producción en sangría del clon RRIM 600; año comercial 2003.T

\begin{tabular}{cccc}
\hline & Tratamiento & Promedio g caucho/árbol/año & Intervalo de confianza g caucho/árbol/año \\
\cline { 1 - 2 } No. & Formulación & & \\
\hline 1 & $\mathrm{~d} / 4$ sin estimulación ${ }^{1}$ & 2816.85 & $3792,85-1840,85^{3}$ \\
2 & $\mathrm{~d} / 4$, ET 2.5\%, 6/y & 4101.11 & $5077,11-3125,11$ \\
3 & $\mathrm{~d} / 4$, ET 2.5\% 7/y & 4446.59 & $5422,59-3470,59$ \\
4 & $\mathrm{~d} / 5$, sin estimulación ${ }^{2}$ & 1752.0 & $2728-776$ \\
5 & $\mathrm{~d} / 5$, ET 3.3\%, $7 / y$ & 3060.27 & $4036,27-2084,27$ \\
6 & $\mathrm{~d} / 5$, ET 3.3\%, 8/y & 3321.98 & $4297,98-2345,98$ \\
\hline
\end{tabular}

1: Tratamiento testigo, en un intervalo de sangría cada cuatro días.

2: Tratamiento testigo, en un intervalo de sangría cada cinco días. d/4: Frecuencia de sangría cada cuatro días. d/5: Frecuencia de sangría cada cinco días. ET $2.5 \%, 6 / y$ : Estimulación con Ethefon al 2.5\%, 6 veces al año.

3: Confiabilidad de $95 \%$.

El promedio y los intervalos de confianza de $\mathrm{g}$ de caucho seco por árbol, obtenidos para cada uno de los tratamientos, se presentan en la Tabla 5. Se observó en la frecuencia de sangría cada cuatro días una producción mayor en $1629.74 \mathrm{~g} /$ caucho/árbol en el tratamiento tres, con aplicación de estimulante respecto del tratamiento testigo. Así mismo, una diferencia de $1569.98 \mathrm{~g} /$ caucho/árbol entre la producción del tratamiento testigo y el tratamiento seis con uso de estimulante y mejor rendimiento en la frecuencia de sangría cada cinco días.

Los contrastes evidencian diferencias significativas en la frecuencia de sangría; la sangría cada cuatro días fue la de mejor rendimiento. El uso del estimulante incide en la producción, tanto en la frecuencia de sangría cada cuatro días como cada cinco días; todos los tratamientos con aplicación de estimulante presentan diferencias significativas frente a los tratamientos sin aplicación de estimulante.

Al comparar los tratamientos dos $(\mathrm{d} / 4$, Eт $2.5 \%$, $6 / y)$ y tres $(\mathrm{d} / 4$, ET $2.5 \%, 7 / \mathrm{y})$ con el cinco $(\mathrm{d} / 5$, ET $3.3 \%, 7 / y)$ y seis $(\mathrm{d} / 5$, ET $3.3 \%, 8 / y)$, que incluían uso de estimulante, se aprecian diferencias significativas; la mayor producción se encontró en la frecuencia de sangría cada cuatro días, con uso de estimulante. Al contrastar los dos tratamientos con aplicación de estimulante de una misma frecuen- cia de sangría, no se hallaron diferencias significativas. Este resultado indica que el aumentar una aplicación anual más de estimulante, tanto en concentración de $2.5 \%$ como de $3.3 \%$, independientemente de la frecuencia de sangrado, no aumentó la producción de g/caucho/árbol.

\section{Clon IAN 873}

La distribución de la producción a través de los diez meses se presenta en la Figura 2. Los resultados del ANAVA para el clon IAN 873 no evidencian diferencias estadísticas entre la producción promedio de los sistemas de sangría evaluados. Los valores de caucho seco por árbol e intervalos de confianza para cada uno de los tratamientos se presentan en la Tabla 6. Es notable que tanto el tratamiento seis, con el mejor rendimiento, y el tratamiento cuatro, con los rendimientos más bajos, se presentan en una frecuencia de sangría cada cinco días, con una diferencia de 1074 g/árbol/año (27.6\%).

\section{Clon $P B 260$}

La Figura 3 presenta el comportamiento de la producción a través de los diez meses. Los resultados del ANAVA para este clon presentan diferencias significativas entre tratamientos; los promedios de gramos de caucho seco e intervalos de confianza por árbol, para cada uno de los tratamientos, se presentan en la Tabla 7. 
La prueba de contrastes entre tratamientos con estimulación dos $(\mathrm{d} / 4$, ET $2.5 \%, 4 / \mathrm{y})$ y tres $(\mathrm{d} / 4$, ET $2.5 \%, 5 / \mathrm{y})$ con el cinco (d/5, ET $2.5 \%, 5 / \mathrm{y})$ y seis (d/5, ET $2.5 \%, 6 / y)$ presentó diferencias significativas. No se observaron diferencias significativas en la producción de tratamientos estimulados en una frecuencia de sangría cada cuatro días; por el contrario, se presentaron diferencias entre los tratamientos cinco y seis, con aplicación de estimulante en una frecuencia de sangría cada cinco días. La sangría cada cuatro días presentó la mayor producción.

Se observó una diferencia en producción de 984.19 g/árbol/año (36.5\%), entre el tratamiento dos con uso de estimulante, sangría cada cuatro días y ma- yor rendimiento, y el tratamiento cuatro sin estimulación, sangría cada cinco días y de menor rendimiento.

\section{Clon GT 1}

La Figura 4 presenta la distribución de la producción de cada uno de los tratamientos evaluados para el clon GT 1 a lo largo de diez meses.

Al realizar el ANAVA se encontraron diferencias significativas entre las producciones promedio obtenidas para los seis tratamientos evaluados en este clon. Los promedios de producción e intervalos de confianza para cada uno de los tratamientos se presentan en la Tabla 8.

Tabla 6. Promedios de producción en sangría del clon IAN 873, año comercial 2003.

\begin{tabular}{|c|c|c|c|}
\hline \multicolumn{2}{|r|}{ Tratamiento } & \multirow{2}{*}{ Promedio g caucho/árbol/año } & \multirow{2}{*}{$\begin{array}{l}\text { Intervalo de confianza } \\
\text { g caucho/árbol/año }\end{array}$} \\
\hline №. & Formulación & & \\
\hline 1 & d/4 sin estimulación 1 & 3267.41 & $4319.41-2215.41^{3}$ \\
\hline 2 & $\mathrm{~d} / 4$, ET $3.3 \%, 7 / y$ & 3412.62 & $4464.62-2360.62$ \\
\hline 3 & $\mathrm{~d} / 4$, ET $3.3 \% 8 / \mathrm{y}$ & 3822.03 & $4874.03-2770.62$ \\
\hline 4 & d/5, sin estimulación ${ }^{2}$ & 2811.21 & $3863.21-1759.21$ \\
\hline 5 & $\mathrm{~d} / 5, \mathrm{ET} 5.0 \%, 7 / \mathrm{y}$ & 3033.44 & $4085.44-1981.44$ \\
\hline 6 & $\mathrm{~d} / 5$, ET $5.0 \%, 8 / \mathrm{y}$ & 3885.85 & $4937.85-2833.85$ \\
\hline
\end{tabular}

1: Tratamiento testigo, en un intervalo de sangría cada cuatro días.

2: Tratamiento testigo, en un intervalo de sangría cada cinco días.

d/4: Frecuencia de sangría cada cuatro días.

d/5: Frecuencia de sangría cada cinco días.

ET 2.5\%, 6/y: Estimulación con Ethefon al 2.5\%, 6 veces al año.

3: Confiabilidad de $95 \%$.

Tabla 7. Promedios de producción en sangría del clon PB 260, año comercial 2003.

\begin{tabular}{cccc}
\hline & Tratamiento & Promedio gr. caucho/árbol/año & $\begin{array}{c}\text { Intervalo de confianza gr. caucho/árbol/ } \\
\text { año }\end{array}$ \\
\cline { 1 - 2 } No. & Formulación & & $20547.25-1441.25^{3}$ \\
\hline 1 & $\mathrm{~d} / 4 \sin$ estimulación ${ }^{3}$ & 1994.25 & $3249.4-2143.4$ \\
2 & $\mathrm{~d} / 4$, ET 2.5\%, 4/y & 2696.40 & $3087.31-1981.31$ \\
3 & $\mathrm{~d} / 4, \mathrm{ET} 2.5 \% 5 / \mathrm{y}$ & 2534.31 & $2265.21-1159.21$ \\
4 & $\mathrm{~d} / 5$, sin estimulación ${ }^{2}$ & 1712.21 & $2503.17-1397.17$ \\
5 & $\mathrm{~d} / 5, \mathrm{ET} 2.5 \%, 5 / \mathrm{y}$ & 1950.17 & $2765.93-1659.93$ \\
\hline
\end{tabular}

1: Tratamiento testigo, en un intervalo de sangría cada cuatro días.

2: Tratamiento testigo, en un intervalo de sangría cada cinco días.

d/4: Frecuencia de sangría cada cuatro días.

d/5: Frecuencia de sangría cada cinco días.

ET 2.5\%, 6/y: Estimulación con Ethefon al 2.5\%, 6 veces al año.

3: Confiabilidad de $95 \%$. 
Tabla 8. Promedios de producción en sangría del clon GT 1, año comercial 2003.

\begin{tabular}{cccc}
\hline & Tratamiento & Promedio g caucho/árbol/año & $\begin{array}{c}\text { Intervalo de confianza g caucho/árbol/ } \\
\text { año }\end{array}$ \\
\cline { 1 - 2 } No. & Formulación & 2194.20 & $2715.2-1673.2^{3}$ \\
\hline 1 & $\mathrm{~d} / 4$, sin estimulación ${ }^{3}$ & 3049.26 & $3570.26-2528.26$ \\
2 & $\mathrm{~d} / 4, \mathrm{ET} 2.5 \%, 4 / \mathrm{y}$ & 3472.68 & $399368-2951.68$ \\
3 & $\mathrm{~d} / 4, \mathrm{ET} 2.5 \% 5 / \mathrm{y}$ & 1742.44 & $2263.44-1221.44$ \\
4 & $\mathrm{~d} / 5$, sin estimulación ${ }^{2}$ & 2747.83 & $3268.83-2226.83$ \\
5 & $\mathrm{~d} / 5, \mathrm{ET} 3.3 \%, 5 / \mathrm{y}$ & 2644.33 & $3165.33-2123.33$ \\
6 & $\mathrm{~d} / 5$, ET 3.3\%, 6/y & & \\
\hline
\end{tabular}

1: Tratamiento testigo, en un intervalo de sangría cada cuatro días.

2: Tratamiento testigo, en un intervalo de sangría cada cinco días.

d/4: Frecuencia de sangría cada cuatro días.

d/5: Frecuencia de sangría cada cinco días.

ET 2.5\%, 6/y: Estimulación con Ethefon al 2.5\%, 6 veces al año

3: Confiabilidad de $95 \%$.

Los contrastes muestran diferencias entre los tratamientos dos (d/4, ET $2.5 \%, 4 / y)$ y tres $(\mathrm{d} / 4$, Ет $2.5 \%$, $5 / \mathrm{y})$ con los tratamientos cinco (d/5, ET $3.3 \%, 5 / \mathrm{y})$ y seis $(\mathrm{d} / 5$, Eт $3.3 \%, 6 / y)$ que incluían estimulación. No se observaron diferencias entre los tratamientos estimulados de la frecuencia de sangría cada cinco días, contrario a los tratamientos con uso de estimulante de frecuencia de sangría cada cuatro días; el tratamiento tres, en una frecuencia de sangría cada cuatro días, fue el de mayor producción.

Se observa una diferencia de 1730.24 g/árbol/año $(49.8 \%)$ entre el tratamientos tres, con uso de esti- mulante, sangría cada cuatro días y mayor rendimiento, y el tratamiento cuatro, sin estimulación, sangría cada cinco días y de menor rendimiento.

En los clones RRIM 600, PB 260 y GT 1 los contrastes presentaron diferencias significativas entre el tratamiento uno, sin uso de estimulante y sangrado cada cuatro días, y el tratamiento cuatro, sin aplicación de estimulante sangrado cada cinco días. Para los tres clones la frecuencia de sangría cada cuatro días presentó los mayores rendimientos.

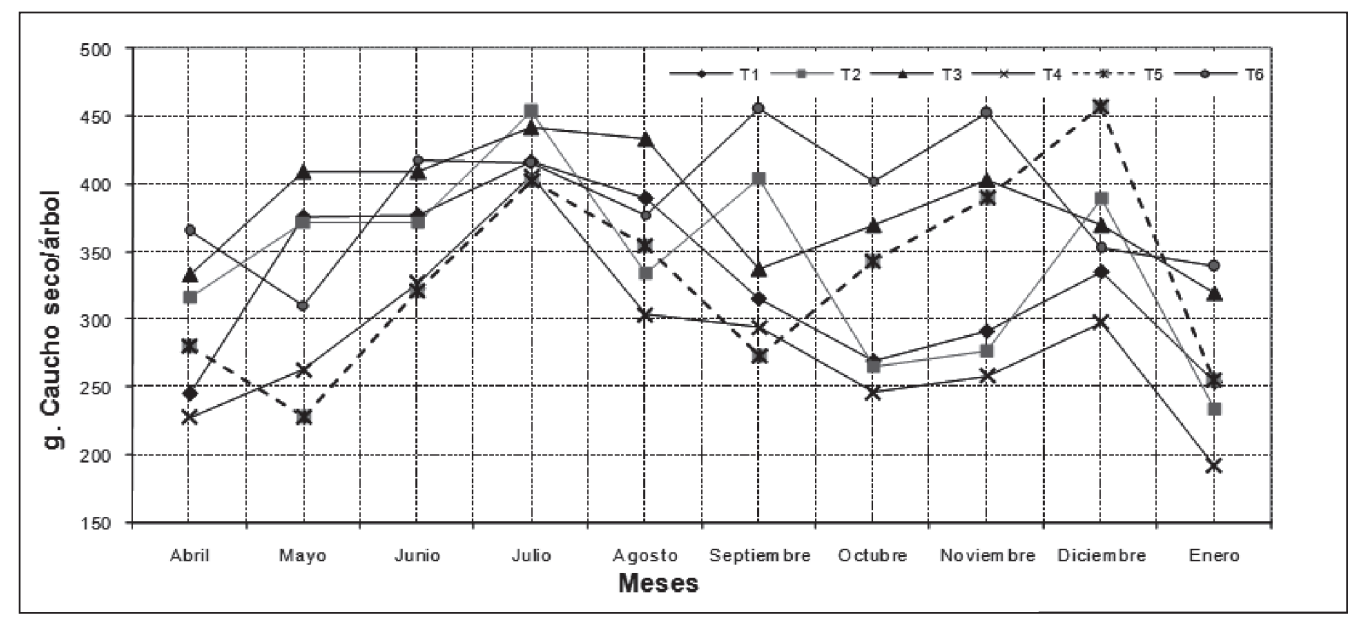

Figura 2. Distribución de la producción promedio mensual de caucho seco por árbol del clon IAN 873 (año comercial de abril 2003 - enero 2004), donde: T1: Testigo en frecuencia d/4, sin aplicación de estimulante; T2: d/4, ET 3.3\%, 7/y; T3: d/4, ET 3.3\%, 8/y; T4: Testigo en frecuencia d/5, sin aplicación de estimulante; T5: d/5, ET $5.0 \%, 7 / y$, y T6: d/5, Eт $5.0 \%, 8 / y$. 


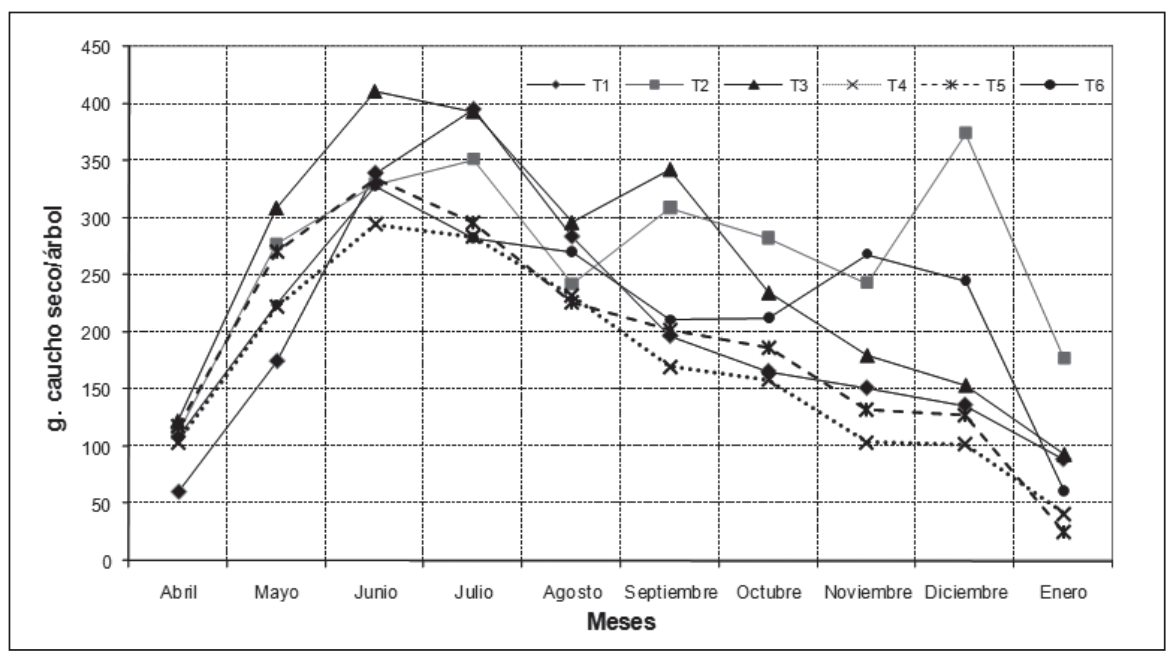

Figura 3. Distribución de la producción promedio mensual de caucho seco por árbol del clon PB 260 (año comercial de abril 2003 - enero 2004), donde: T1: Testigo en frecuencia d/4, sin aplicación de estimulante; T2: d/4, ET 2.5\%, 4/y; T3: d/4, ET 2.5\%, 5/y; T4: Testigo en frecuencia $\mathrm{d} / 5$, sin aplicación de estimulante; T5: d/5, ET 2.5\%, 5/y, y T6: d/5, Eт 2.5\%, 6/y.

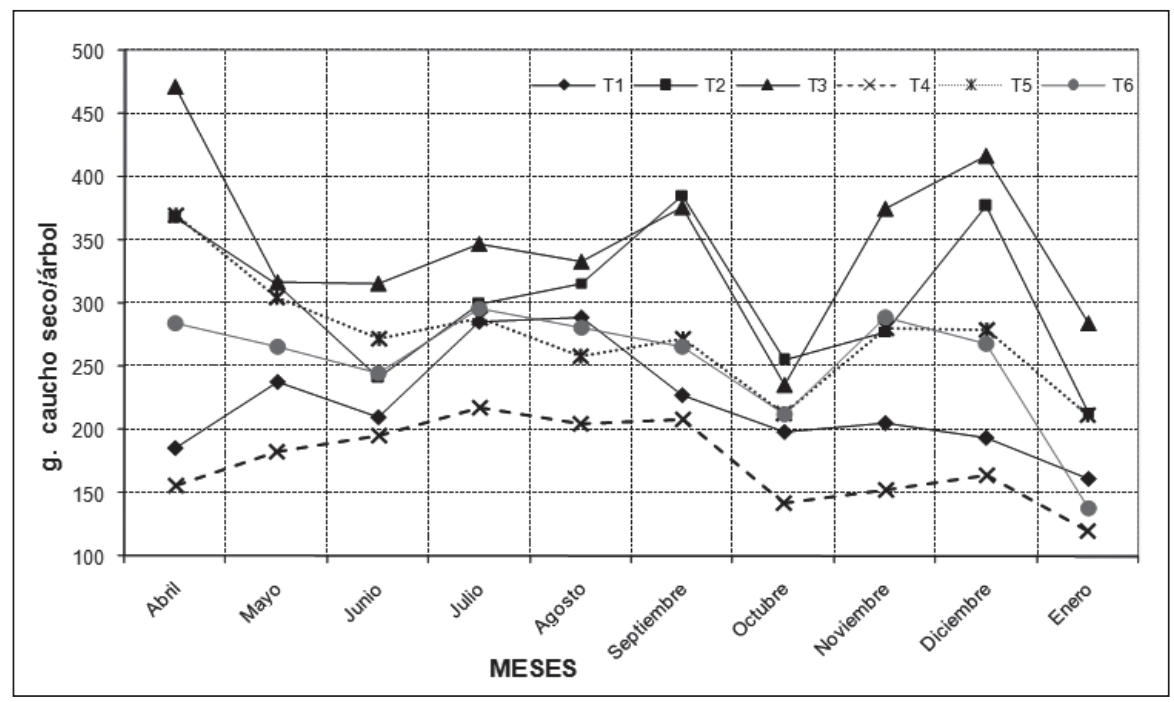

Figura 4. Distribución de la producción promedio mensual de caucho seco por árbol del clon GT 1 (año comercial de abril 2003 - enero 2004), donde: T1: Testigo en frecuencia d/4, sin aplicación de estimulante; T2: d/4, ET 2.5\%, 4/y; T3: d/4, ET 2.5\%, 5/y; T4: Testigo en frecuencia d/5, sin aplicación de estimulante; T5: d/5, ET 3.3\%, 5/y, y T6: d/5, ET 3.3\%, 6/y.

\section{DISCUSIÓN}

Los valores de producción obtenidos en este estudio para el clon RRIM 600 son similares a los obtenidos en estudios realizados por Goncalves et al. (2000) en São Paulo, Brasil, en el municipio de Indiana, donde este clon en el tercer año de sangría presentó producciones promedio de $4536 \mathrm{~g} /$ árbol/año. Con respecto al clon IAN 873, en tercer año de sangría, los autores en este estudio reportan producciones de $4097 \mathrm{~g} /$ árbol/año, cuyo valor es superior en 209 g caucho/árbol/año a los resultados obtenidos en este estudio.

Goncalves et al. (2001a) reportan valores inferiores a los promedios de producción obtenidos en este estudio en $1337 \mathrm{~g} /$ caucho/árbol/año y 380 g/caucho/árbol/año para los clones IAN 873 y RRIM 
600 , respectivamente, en tercer año de sangría en el estado de São Paulo, Brasil, en el municipio de Votuporanga, donde se obtuvo una producción promedio de $2550 \mathrm{~g} /$ caucho/árbol para el clon IAN 873 y una producción promedio de 4060 g/caucho/árbol para el clon RRIM 600 .

De acuerdo con los resultados obtenidos bajo las condiciones de este trabajo se pueden estimar, en un tercer año de sangría, producciones promedio por árbol de $4.5( \pm 0.976) \mathrm{kg}$, con sangría cada cuatro días y siete aplicaciones anuales de estimulante a una concentración de $2.5 \%$, y $3.8( \pm 1.052) \mathrm{kg}$ de caucho seco por árbol al año, con sangría cada cuatro días, ocho aplicaciones anuales de estimulante en una concentración de $3.3 \%$ para los clones RRIM 600 e IAN 873 , respectivamente.

Los resultados obtenidos para el clon GT 1 en las condiciones en que se realizó éste trabajo son superiores en $295 \mathrm{~g} /$ caucho/árbol/año a los reportados por Pereira et al. (2001) en su estudio en el cerrado brasilero, en los municipios de Barro Alto y Goianésia en el estado de Goiás, donde reportan una producción promedio de $3177 \mathrm{~g} /$ árbol/año en el primer año de sangría. Goncalves et al. (2000) reportan valores de inferiores en 342 g/caucho/árbol/año para este clon, que obtuvo una producción promedio de $3130 \mathrm{~g} / \mathrm{caucho/árbol/año} \mathrm{en} \mathrm{su} \mathrm{primer}$ año de sangría.

En las condiciones de este estudio se estima, en primer año de sangría, producciones de caucho seco aproximadas de $2.7( \pm 0.553) \mathrm{kg} /$ árbol/año, con sangría cada cuatro días y el uso de estimulante cuatro veces por año en una concentración de $2.5 \%$, y 3.5 ( \pm 0.521$) \mathrm{kg} /$ árbol/año con sangría cada cuatro días y uso de estimulante cinco veces por año en una concentración de $2.5 \%$ para los clones PB 260 у GT 1, respectivamente.

Basado en los resultados obtenidos para el clon RRIM 600 y a través de un cálculo del promedio de kilogramos de caucho por hectárea al año, con 400 árboles en sangría, se puede estimar una producción de $1780 \mathrm{~kg} / \mathrm{ha}$. Estos estimativos son superiores a los reportados en el estado de São Paulo por Goncalves et al. (2001b), quienes presentan un rendimiento de $1545 \mathrm{~kg} / \mathrm{ha}$, y por Chan (1984) en la península malaya, donde para el clon RRIM 600 en tercer año de sangría promedia una producción anual de $1290 \mathrm{~kg} / \mathrm{ha}$. Así mismo, se reporta una producción promedio anual de $1140 \mathrm{~kg} / \mathrm{ha}$ de caucho seco para el clon PB 260 en su primer año de sangría; el cálculo de producción anual promedio por hectárea para este clon con los resultados obtenidos en este estudio y un hipotético de 250 árboles/ha resulta en $675 \mathrm{k} / \mathrm{ha}$.

Una estimación de $868 \mathrm{~kg}$ de caucho por ha/año para el clon GT 1, con los resultados obtenidos en este estudio y 250 árboles en sangría, son levemente superiores al reporte de $776 \mathrm{~kg}$ por ha/año efectuado Goncalves et al. (2002) en ensayos de pequeña escala en Pindorama, en el estado de São Paulo en Brasil.

Teniendo en cuenta lo observado en los clones RRIM 600 у Рв 260, en los que no se encontraron diferencias en los tratamientos que incluían la misma frecuencia de sangría cada cuatro días, una misma concentración de estimulante y que tan sólo se diferenciaban en una aplicación anual más de estimulante, existe la posibilidad de plantear la evaluación de tratamientos que contemplen una frecuencias cada cuatros días y una concertación de estimulante de $2.5 \%$ y cuya aplicación se realice hasta un máximo de ocho veces por año; esto debido a su carácter de clones con baja producción de azúcares como sugieren Moreno et al. (2003).

Cabe anotar que los resultados presentados en este trabajo constituyen tan sólo una parte preliminar del proceso de recomendación del mejor sistema de sangría para cada uno de los clones, ya que este tipo de conceptos se emiten después de un extenso periodo de evaluación (mínimo 5 años), que involucra parámetros como el número de árboles con secamiento del panel y en lo posible parámetros fisiológicos asociados a la producción conocida como "técnica de diagnóstico látex".

\section{CONCLUSIONES}

El mejor sistema de sangría para el tercer año de aprovechamiento del clon RRIM 600 consta de una frecuencia de sangrado cada cuatro días, con siete 
aplicaciones anuales de agente estimulante en una concentración de $2.5 \%$. Con una notación internacional $1 / 2 \mathrm{~S}, \mathrm{~d} / 4,6 \mathrm{~d} / 7,10 \mathrm{~m} / 12$, ET $2.5 \%$, Pa $2 / 1$, $7 / \mathrm{y}$.

Debido a que no hubo diferencias entre valores promedio de producción de caucho seco por árbol, en los diferentes sistemas evaluados para el clon IAN 873, se puede sugerir par el manejo de este clon una frecuencia de sangría cada cinco días, ya que esta practica disminuye costos de mano de obra. Así, un sistema de sangría favorable para este clon constaría de una frecuencia de sangrado cada cinco días, con siete aplicaciones anuales de agente estimulante en una concentración del 5\%, el cual se notaría internacionalmente así: $1 / 2 \mathrm{~S}, \mathrm{~d} / 5,6 \mathrm{~d} / 7$, $10 \mathrm{~m} / 12$, Ет $5.0 \%$, Pa 2/1, 7/y.

Para el clon PB 260 en un primer año de sangría, se sugiere una frecuencia de sangría cada cuatro días con siete aplicaciones anuales de agente estimulante en una concentración de $2.5 \%$, el cual se nota internacionalmente así: $1 / 2 \mathrm{~S}, \mathrm{~d} / 4,6 \mathrm{~d} / 7,10 \mathrm{~m} / 12$, Ет 2.5\%, Pa 2/1, 7/y. Esta frecuencia ofrece los mejores resultados de producción en $\mathrm{g}$ de caucho seco por árbol.

El clon GT 1, en un primer año de aprovechamiento, presentó los mejores niveles de producción de caucho seco por árbol, en sistema de sangría, con una frecuencia de sangrado cada cuatro días, con cinco aplicaciones anuales de agente estimulante en una concentración de $2.5 \%$. Su notación internacional es: $1 / 2 \mathrm{~S}, \mathrm{~d} / 4,6 \mathrm{~d} / 7,10 \mathrm{~m} / 12$, ет $2.5 \%, \mathrm{~Pa} 2 / 1,5 / \mathrm{y}$.

\section{AGRADECIMIENTOS}

Los autores expresan sus agradecimientos al ingeniero Carlos Alberto Pérez y al personal técnico y administrativo de la empresa MAVALLE S.A. por su valiosa contribución y apoyo. De igual forma, al Ministerio de Agricultura y Desarrollo Rural por el apoyo financiero (Contrato 068/2002) para la realización de este proyecto.

\section{REFERENCIAS BIBLIOGRÁFICAS}

Compagnon, P. 1998. El caucho natural: biología, cultivo, producción. Edición en español. Consejo Mexicano del Hule y CIRAD. Ciudad de México. Pg 701.

Chang, W. H. 1997. Perfomance of clone Рв 260 in a large plantation group in peninsular Malaysia REVIEWED. The planter 73 (8): 403-418.

Corporación Nacional de Investigación y Fomento Forestal (CONIF) y Ministerio de Agricultura y Desarrollo Rural. 1997. Plan de Investigación en caucho natural. Serie técnica 38, Bogotá.

D’Auzac, J., Jacob, J. L. \& H. Chrestin. 1989. Physiology of Rubber Tree Latex. CRC Press. Boca Raton. Pg. 470.

Doree, J. F. 1998. Rapport Annuel Agricole 199596-97. Departement des Techniques Agricoles. Matto Groso, Brasil. Pg. 45.

Gonçalves, P. de S., S. R. De Souza, P. A. Brioschi, A. V. Castro, F. May, A. Sanches \& C. Alarcon. 2000. Efeito da freqüência de sangria e estimulação no desempenho produtivo e econômico de clones de seringueira. Pesquisa Agropecuária Brasileira 35 (6): 1081-1091.

Gonçalves, P. de S., N. Bortoletto, E. L. Furtado, R. E. Sambugaro, O. C. Bataglia. 2001a. Desempenho de clones de seringueira da série IAC 300 selecionados para a região noroeste do Estado de São Paulo. Pesquisa Agropecuária Brasileira 36 (4): 589-599.

Gonçalves, P. de S., N. Bortoletto, R. Sambugaro, E. L. Furtado, O. C. Bataglia, A. O. Aldo \& G. J. Gentil. 2001b. Desempenho de clones de seringueira de origem amazônica no planalto do Estado de São Paulo. Pesquisa Agropecuária Brasileira 36 (12): 1469-1477.

Gonçalves, P. de S., M. A. Mello, L. Furtado, E. L. Sambugaro, R. E. Ottati, E. L. Altino, A. O. Aldo \& G. L. Gentil. 2002. Desempenho de clones de seringueira da série IAC 300 na re- 
gião do planalto de São Paulo. Pesquisa Agropecuária Brasileira 37 (2): 131-138.

Instituto de Biotecnología de la Universidad Nacional de Colombia. 2005. Ajuste y desarrollo tecnológico en los procesos de extracción y beneficio de caucho natural (Hevea brasiliensis). Informe final presentado al Ministerio de Agricultura y Desarrollo Rural. Proyecto 038/2003 MADR-IBUN. Bogotá.

López, J. A. 2003. Comportamiento del caucho Hevea barsiliensis en la zona cafetera. Fitotecnia Colombiana 3 (1): 1-8.

Ministerio de Agricultura y Desarrollo Rural. 2008. Bulles: cadena productiva del caucho y su industria. Informe presentado por secretaria técnica a MADR. Bogotá.
Moreno, R. M., M. Ferreira, P. de S. Goncalves \& L. H. Capparelli Matosso. 2003. Avaliacao de látex de borracha natural de clones de seringueira no Estado de Sao Paulo. Pesquisa Agropecuária Brasileira 38 (5): 583-590.

Pereira, A. V., P. B. Carvalho \& B. F. Canuto. 2001. Desempenho de clones de seringueira sob diferentes sistemas de sangria. Pesquisa Agropecuária Brasileira 36 (1): 389-399.

Statistical Analysis System. 1999. SAS User's Guide: Statistics (Version 8.0 Ed.). SAS Inst. Inc. Cary, NC.

Torres, C. 1984. El caucho hevea en Colombia. Instituto Colombiano de la Reforma Agraria. Boletín Técnico No. 18. Florencia, Colombia. Pg 87. 